Rev. Int. Contam. Ambie. 33 (1) 65-73, 2017

DOI: 10.20937/RICA.2017.33.01.06

\title{
GENERACIÓN DE POLVOS DE MADERA EN TALLERES DE LA ESCUELA DE LAUDERÍA DEL INSTITUTO NACIONAL DE BELLAS ARTES Y LITERATURA, MÉXICO
}

\author{
Joaquín Antonio QUIROZ CARRANZA ${ }^{1 *}$, Abraham Marcelino VIDAL LIMÓN² y \\ Jesús Alejandro TORRES TORRES 3
}

${ }^{1}$ Facultad de Ciencias Naturales, Universidad Autónoma de Querétaro. Avenida de las Ciencias sin número, Juriquilla, Santa Rosa Jáuregui, Querétaro, México, C. P. 76230

${ }^{2}$ Instituto Potosino de Investigación Científica y Tecnológica. Camino a la Presa de San José 2055, Lomas 4ta. sección, San Luis Potosí, México, C. P. 78216

${ }^{3}$ Escuela de Laudería, Instituto Nacional de Bellas Artes y Literatura. Avenida Miguel Hidalgo 20, Centro Histórico, Santiago de Querétaro, Querétaro, México, C. P. 76000

*Autor para correspondencia: citlallicg@yahoo.com

(Recibido enero 2016; aceptado junio 2016)

Palabras clave: exposición ocupacional, partículas de madera en interiores, elaboración de violines

\section{RESUMEN}

El objetivo del presente trabajo fue identificar el riesgo por la exposición a polvos de madera en los talleres de construcción de instrumentos musicales de cuerda frotada, de la Escuela de Laudería del Instituto Nacional de Bellas Artes y Literatura, México, pues dicha exposición está asociada con enfermedades alérgicas y no alérgicas como dermatitis, conjuntivitis-rinitis, asma, alveolitis alérgica extrínseca y cáncer, entre otras. Se analizó la cantidad de partículas de madera iguales o menores a $2.5 \mu \mathrm{m}$ presentes en el aire del interior de tres talleres de construcción de instrumentos. Asimismo, se realizaron dos análisis gravimétricos: uno por cuatro días en ciclos de $24 \mathrm{~h}$ en tres talleres y otro durante 10 días en ciclos de $4 \mathrm{~h}$ en tres talleres. Los resultados gravimétricos evidencian que la concentración de polvos de madera en el aire del interior de los talleres estudiados supera las normas nacionales, así como algunas internacionales. Lo anterior se manifiesta con malestares y síntomas expresados por los usuarios de los talleres de construcción de instrumentos musicales. Adicionalmente, se realizó una encuesta sobre la percepción pública de la contaminación del aire del interior con énfasis en los efectos de los polvos de madera sobre la salud, que fue aplicada a los alumnos y profesores de la institución.

Key words: occupational exposure, indoor wood dust, violin maker

\begin{abstract}
The aim of this work was to identify the risk of exposure to wood dust in workshops of musical instruments, at Instituto Nacional de Bellas Artes y Literatura's Violin Making School, Mexico, since such exposure is associated to allergic and non-allergic diseases, such as dermatitis, conjunctivitis-rhinitis, asthma, extrinsic allergic alveolitis and cancer, among others. The amount of particles equal to or less than $2.5 \mu \mathrm{m}$ present in the air of three workshops of musical instruments was analyzed. Two gravimetric
\end{abstract}


analyses were made: one for four days in twenty-four-hour cycles in three workshops, and the other for ten days in four-hour cycles in three workshops. Additionally, a survey on public perception of indoor air pollution with an emphasis on the effects of wood dust on health was performed. This survey was applied to students and professors of the institution. The gravimetric results showed that the concentration of wood dust on indoor air of the studied workshops exceeds national and several international standards, evidenced through malaise and symptoms expressed by users of the workshops of musical instruments.

\section{INTRODUCCIÓN}

Durante la construcción de instrumentos musicales de cuerda frotada, como son violines, violas, violoncellos y contrabajos, los alumnos y profesores de la Escuela de Laudería del Instituto Nacional de Bellas Artes y Literatura (INBAL), realizan una serie de procesos que se agrupan en dos etapas: de preparación y de construcción. En ambas etapas se generan residuos de diverso tamaño como virutas, aserrín y polvos, que entran en contacto con los constructores por vía dérmica, ocular y respiratoria. A manera de ejemplo se puede mencionar que al concluirse la construcción de un violín clásico, éste contiene únicamente el $15 \%$ de la madera inicial, lo que implica que el $85 \%$ de la madera restante se convirtió en viruta, aserrín y partículas de diverso tamaño.

Existen estudios sobre la generación de polvos de madera y sus efectos sobre la salud humana como los de Teschke et al. (1999), Schlünssen et al. (2001), Meo (2004), Husgafvel-Pursiainen y Savolainen (2006), Kauppinen et al. (2006). Bornholdt (2008). Sin embargo, estos estudios se han realizado en países de Europa y Norteamérica. En México no se ha estudiado la cantidad de polvos de madera generados durante la construcción de instrumentos musicales de cuerda frotada y sus efectos sobre la salud humana.

El tamaño, la composición química y la abundancia de los polvos de madera generados durante la construcción de instrumentos musicales, son características que se deben estudiar debido a que la permanencia de las partículas de madera con un diámetro de $1 \mu \mathrm{m}$ del interior es de $0.003 \mathrm{~cm} / \mathrm{s}$, lo que permite que éstas entren en contacto con los constructores de instrumentos por vía dérmica, ingesta e inhalación. Lo anterior provoca diversas patologías como asma no alérgica, rinitis, bronquitis crónica, obstrucción crónica y alveolitis alérgica extrínseca (Kenny et al. 1999, Douwes et al. 2003).

Las partículas de madera afectan la salud humana no sólo por las sustancias naturales que contiene este material, sino también por otras que se incorporan durante su manejo en plantaciones, aserraderos, almacenes y carpinterías. Algunas de estas otras sustancias son los conservadores y plaguicidas, así como partículas biológico-infecciosas, entre otras.

Por otro lado, los polvos de madera tienen una composición química que puede ser perjudicial para la salud humana. Está constituida fundamentalmente de celulosa, hemicelulosa, lignina y extraíbles. En el caso de estos últimos tenemos por ejemplo: terpenos, ácidos grasos, compuestos aromáticos, aceites volátiles, taninos, flavonoides, quinonas, lignasas, resinas, alcoholes y alcaloides (Echenique-Manrique y Robles 1993, Teschke et al. 1999, Meo 2004).

El efecto a la salud producido por los polvos de la madera depende de la especie de árbol, del periodo de exposición, de la recurrencia y de los compuestos presentes en la madera. Por ejemplo, la caoba (Swietenia macrophylla) presenta antotecol y dimetoxibenzoquinona. Especies del género Dalbergia y Diospyros poseen quinonas, en tanto que los pinos contienen colofonía (Ideara 2014).

Gioffre et al. (2012) y Magagnotti et al. (2013) mencionan que los polvos de madera dura han sido caracterizados como carcinogénicos. Según Fengel y Wegener (1989) el arce (Acer pseudoplatanus) tiene $2.5 \%$ de extractos etanol-benceno y sus polvos causan dermatitis, mientras que los del pinabete ( $P i$ cea abies) provocan dermatitis, conjuntivitis-rinitis, asma, alveolitis alérgica extrínseca y los del ébano (Diospyros spp.) dermatitis, conjuntivitis-rinitis y asma (Ideara 2014). Goldsmith y Shy (1988) reunieron la información generada por más de 15 autores sobre los efectos de los polvos de madera de diversas especies sobre la salud humana (Cuadro I).

En empresas donde se trabaja la madera, el material particulado ( $\mathrm{PM}$, por sus siglas en inglés) además de los polvos de madera, está constituido por otras partículas dañinas como conservadores químicos, pegamentos, barnices, residuos biológicos, polvos de metales y de polímeros. Los polvos de la madera son partículas pequeñas secas y sólidas con un diámetro aerodinámico entre 1 y $100 \mu \mathrm{m}$ (Kauppinen et al. 2006). Dado su bajo peso pueden permanecer suspendidas en el aire por diversos periodos de tiempo, 
CUADRO I. EFECTOS DE POLVOS DE MADERA SOBRE LA SALUD HUMANA

\begin{tabular}{|c|c|c|}
\hline Especie & $\begin{array}{l}\text { Duración de la } \\
\text { exposición }\end{array}$ & Efectos observados \\
\hline $\begin{array}{l}\text { Pterocarpus angolensis, Chlorophora excelsa, Milletia } \\
\text { stuhlmanni, Thuja plicata, Lovoa klaineana }\end{array}$ & 3 meses & Asma, rinitis y disnea \\
\hline Sequoia sempervirens & $2-3$ años & Asma, disnea y sibilancias \\
\hline Quercus sp., Swietenia sp. y Cedrela sp. & 3 meses & Asma, rinitis y opresión del pecho \\
\hline Cedrus libani & 1 año & Asma y disnea \\
\hline Dalbergia retusa & $3-18$ meses & Irritación de las vías aéreas superiores \\
\hline Lucuma sp. & Días a un año & Asma con disnea nocturna \\
\hline Gonystylus bancanus & No establecido & Hipersensibilidad con disnea, tos y sudoración \\
\hline $\begin{array}{l}\text { Microberlinia sp. Tectonia grandis, } \\
\text { Aucoumea klaineana, Aningeria sp. }\end{array}$ & 5 meses & Asma o neumonitis con tos, disnea y bronquitis \\
\hline Juglans olanchana & No establecido & Rinorrea, tos, sibilancias y disnea \\
\hline Triplochiton scleroxylon & $1-7$ años & Rinitis, sibilancias, disnea, estornundos, prurito nasal. \\
\hline
\end{tabular}

Fuente: Goldsmith y Shy (1988)

pueden sedimentarse y volver a re-suspenderse por causa del viento o el tránsito del personal que allí labora. La permanencia en el aire de polvos de madera mayores de $100 \mu \mathrm{m}$ se estima en $2.62 \times 10^{-1} \mathrm{~cm} / \mathrm{s}$, mientras que aquellas con un diámetro de $1 \mu \mathrm{m}$ es de $3.06 \times 10^{-3} \mathrm{~cm} / \mathrm{s}$ (Kochevar 2006).

Las partículas atmosféricas se clasifican en: 1) fracción inhalable, con un diámetro de entre 100 y $50 \mu \mathrm{m}\left(\mathrm{PM}_{100}\right.$ y $\left.\mathrm{PM}_{50}\right)$, estas partículas tras ingresar al organismo se depositan en las fosas nasales y la laringe (Ideara 2014), 2) fracción torácica, con un diámetro entre 10 y $2.5 \mu \mathrm{m}\left(\mathrm{PM}_{10}\right.$ y $\left.\mathrm{PM}_{2.5}\right)$, que penetran en las vías respiratorias, son retenidas en las vías aéreas superiores y expulsadas por el organismo junto con las mucosidades, los daños potenciales pueden ser sobre la piel y los ojos y 3) fracción respirable, con un diámetro aerodinámico entre 2.5 y $1 \mu \mathrm{m}\left(\mathrm{PM}_{2.5}\right.$ y $\mathrm{PM}_{1.0}$ ), ingresan más allá de los alveolos hasta la región de intercambio de gases de los pulmones y se incorporan al torrente sanguíneo (Magagnotti et al. 2013). Las $\mathrm{PM}_{2.5}$ y $\mathrm{PM}_{1.0}$ son peligrosas porque facilitan el transporte y la penetración al organismo de otras sustancias que se adsorben en sus cavidades, poros y superficies (Tzintzun et al. 2005).

Para contribuir al entendimiento de los fenómenos relacionados con partículas atmosféricas, sobre todo de ambientes de interiores, además de los análisis gravimétricos es necesario conocer la percepción de las personas que viven un fenómeno de contaminación ambiental para analizar sus propuestas e involucrarlos en el manejo de los riesgos (Catalán-Vázquez y Jarillo-Soto 2010).

En este trabajo se analizó, mediante gravimetría, la cantidad de polvos de madera menores a $2.5 \mu \mathrm{m}$ de diámetro presentes en el aire del interior de tres talleres de construcción de instrumentos musicales de la Escuela de Laudería del INBAL, ubicado en Querétaro, México. Además, se indagó sobre la percepción de alumnos y profesores de los talleres de construcción de instrumentos musicales de esta institución respecto a los efectos de los polvos de madera sobre su salud, a través de un cuestionario estructurado. El estudio se realizó con la finalidad de estimar los efectos potenciales de los polvos de madera sobre la salud de profesores y alumnos de la institución para discutir la necesidad de aplicar medidas de prevención personales e institucionales que minimicen la cantidad de polvos de madera en el aire del interior de los talleres de construcción que sirva de base en otros emprendimientos del ramo.

\section{MATERIALES Y MÉTODOS}

\section{Sitio de estudio}

La Escuela de Laudería del INBAL se fundó en 1987 y en 1992 se trasladó a Querétaro. A partir de 2005 su sede es un inmueble colonial ubicado en la calle Hidalgo número 20, Centro, Santiago de Querétaro (Lavalle 2015). Originalmente el inmueble 
mencionado tenía uso habitacional y estaba ocupado por una familia. Cuando la Escuela de Laudería se instaló en el inmueble citado, se transformaron tres de las habitaciones en talleres de construcción de instrumentos de cuerda frotada. Cada taller reúne a seis alumnos, los que construyen sus instrumentos y un profesor coordina las sesiones. Las actividades de construcción que generan polvos de madera se realizan de lunes a viernes de $7 \mathrm{a} 11 \mathrm{~h}$.

\section{Análisis gravimétrico}

El análisis gravimétrico de los polvos de madera en el aire de interiores se llevó a cabo en dos etapas: La primera fue un muestreo diagnóstico, el cual se realizó en los tres talleres de construcción de instrumentos musicales por cuatro días en ciclos de $24 \mathrm{~h}$. La segunda etapa se realizó en los tres talleres, por diez días cada uno, en ciclos de $4 \mathrm{~h}$, entre las 7 am y las $11 \mathrm{am}$, que es el horario en que se realiza la construcción de instrumentos musicales de cuerda frotada. Para capturar los polvos se usó un muestreador táctico para partículas suspendidas MiniVol (Airmetrics) con un impactador para retener únicamente partículas con diámetros iguales o menores a $2.5 \mu \mathrm{m}$. Los muestreos se realizaron con un flujo de aire de $5 \mathrm{~L}$ por minuto, durante abril y mayo de 2015.

Los polvos de madera se retuvieron con filtros de fibra de vidrio de $47 \mathrm{~mm}$ de diámetro y con un poro de 0.7 micras de diámetro (Millipore, MA, USA) (Magagnotti et al. 2013). Los polvos se estabilizaron por $48 \mathrm{~h}$ en horno eléctrico, a una temperatura de $26^{\circ} \mathrm{C}$ y se pesaron en una balanza analítica de cuatro decimales (Ohaus Adventurer). Una vez realizado el muestreo, los filtros se estabilizaron y se pesaron bajo las mismas condiciones (Gioffre et al. 2012). La concentración de polvos de madera en el filtro fue obtenida usando las fórmulas 1 y 2 (Airmetrics 2012).

$\mathrm{V}=\frac{60_{\mathrm{min} / \mathrm{hr}} \times \mathrm{Lpm} \times \mathrm{T}_{\mathrm{h}}}{1000 \mathrm{~L} / \mathrm{m}^{3}}$

Donde $\mathrm{V}$ es el volumen en $\mathrm{m}^{3}$, $\mathrm{Lpm}$ es el flujo de operación del equipo (equivale a $5 \mathrm{~L} / \mathrm{min}$ ) y $\mathrm{T}_{\mathrm{h}}$ es el periodo de muestreo en horas.

$\mathrm{PM}_{\mathrm{act}}=\frac{\mathrm{M}_{\mathrm{PM}}}{\mathrm{V}}$

Donde $\mathrm{PM}_{\text {act }}$ es la concentración actual del material particulado en $\mu \mathrm{g} / \mathrm{m}^{3}, \mathrm{~V}_{\text {act }}$ el volumen en $\mathrm{m}^{3}$ y $\mathrm{M}_{\mathrm{PM}}$ es la masa de partículas colectadas en el filtro en $\mu \mathrm{g}$.

\section{Encuesta de percepción}

Se utilizó una encuesta con 11 reactivos de opción múltiple (ver encuesta), para conocer la percepción sobre la presencia de polvos de madera en el aire del interior y su efecto en la salud humana. Este cuestionario se aplicó a la población docente y a 20 alumnos de los tres talleres de construcción de instrumentos musicales de cuerda frotada. De acuerdo con Catalán-Vázquez y Jarillo-Soto (2010), se consideró lo que los usuarios sienten, opinan y saben sobre los riesgos ocasionados por la contaminación del aire de interiores.

\section{Encuesta}

Edad:

Sexo: Masculino ( ) Femenino ( ).

Posición académica: ( ) Estudiante ( ) Profesor

1. ¿Durante cuántos años has trabajado la madera?

2. Menciona el nombre común de las maderas que has usado en el último año. Anota su frecuencia de uso, mencionando el tipo de residuo que libera más ampliamente (polvo, aserrín, viruta).

3. ¿Cuántas horas al día trabajas la madera?

4. ¿Has padecido alguna molestia en la piel, ojos, nariz o garganta en el último año?

\section{Si ( ) No ( ), si es no, pasa a la pregunta 7.}

5. ¿Cuáles son las molestias que has padecido en el último año?

$(1=$ mayor frecuencia, $2=$ frecuencia regular, $3=$ poco frecuente, $4=$ ocasional)

Ojos: ( ) Conjuntivitis ( ) Irritación ( ) Escozor () Enrojecimiento

Nariz: ( ) Irritación ( ) Rinitis ( ) Flujo nasal ( ) Estornudos frecuentes

Garganta: ( ) Irritación ( ) Sequedad ( ) Tos ( ) Asma

Piel: ( ) Dermatitis ( ) Irritación ( ) Escozor ( ) Erupciones

6. ¿Consideras que las molestias se deben al contacto con los polvos de madera?

Si ( ) No ( ) Explica ¿por qué?

7. ¿Cuáles son las principales situaciones de riesgo que se presentan durante el trabajo con la madera? $(1=$ mayor, $5=$ menor $)$ 
( ) Inhalación de polvos ( ) Inhalación de vapores químicos ( ) Ruido

() Contacto dérmico con polvos ( ) Contacto dérmico con sustancias químicas

( ) Daños en manos y brazos ( ) Ninguna ( ) Otra, especificar

8. Actualmente ¿usas equipo de protección contra polvos de madera?

Si ( ) No ( ) Explica ¿por qué?

9. ¿Qué equipo de protección contra polvos de madera usas en tus áreas de trabajo?

( ) Ropa de trabajo ( ) Mascarilla () Guantes de seguridad ( ) Tapones para oídos ( ) Extractor-purificador de aire ( ) Lentes de protección ( ) Otro

10. ¿Cuál de las actividades listadas consideras que provocan mayor cantidad de aserrín y polvos de madera? $(1$ = mayor cantidad, 2 = cantidad regular, $3=$ poca cantidad)

( ) Tallado ( ) Excavado ( ) Cortado ( ) Cepillado ( ) Lijado ( ) Pulido ( ) Desbastado

11. ¿Te has realizado alguno de los análisis clínicos listados, durante el último año?

() Medición de la capacidad auditiva ( ) Oftalmología ( ) Exploración cutánea

( ) Radiografía del tórax ( ) Análisis general.

\section{RESULTADOS}

Los resultados permitieron describir la cantidad de polvos presentes en el aire del interior de los talleres de construcción de instrumentos musicales y la percepción de los usuarios sobre los efectos en la salud humana de los polvos de madera generados durante la construcción de instrumentos musicales.

\section{Análisis gravimétrico}

Durante la construcción de instrumentos musicales de cuerda frotada en los talleres de la Escuela de Laudería del INBAL se generan diversos tipos de partículas atmosféricas debido a los materiales y procesos que allí se desarrollan. Algunos ejemplos son: polvos poliméricos derivados de la elaboración de los moldes con estireno, polvos metálicos durante el afilado de la navajas y los principales por el volumen de generación, son los polvos de la madera.

Las maderas más frecuentemente utilizadas durante la etapa de preparación son: El maple (Acer rubrum L.), el cedro (Cedrela odorata) y el pino (Pinus sp.), así como triplay y fibra de densidad media (MDF, por sus siglas en inglés). Durante la etapa de construcción las maderas más utilizadas son: el arce (Acer pseudoplatanus), el pinabete (Picea abies), el maple (Acer rubrum L.) y el ébano (Diospyros crassiflora) (Cuadro II).

La concentración promedio de polvos de madera, obtenidos durante el muestreo diagnóstico en los tres talleres de la Escuela de Laudería del INBAL, fue de $2.0 \pm 0.52 \mathrm{mg} / \mathrm{m}^{3}$ en $24 \mathrm{~h}$. Esto supera el límite de la norma francesa y canadiense que es de $1.0 \mathrm{mg} / \mathrm{m}^{3}$.

La concentración de polvos de madera en el taller 2 fue de $2.16 \mathrm{mg} / \mathrm{m}^{3}$, resultando superior al límite Danés y Sueco que es de $2.0 \mathrm{mg} / \mathrm{m}^{3}$, así como del Francés y Canadiense que es de $1.0 \mathrm{mg} / \mathrm{m}^{3}$. Cabe mencionar que ninguno de los tres sobrepasa el límite Finlandés y Español de los $5 \mathrm{mg} / \mathrm{m}^{3}$ (Cuadro III).

La concentración promedio de polvos de madera obtenidos durante el muestreo definitivo fue de $0.72 \pm 0.10 \mathrm{mg} / \mathrm{m}^{3}$ en cuatro horas y con una estimación

CUADRO II. CARACTERÍSTICAS DE LAS MADERAS USADAS DURANTE LA CONSTRUCCIÓN DE INSTRUMENTOS MUSICALES DE CUERDA FROTADA

\begin{tabular}{|c|c|c|c|c|}
\hline Nombre común & Nombre científico & Familia botánica & Distribución & Densidad de la madera $\left(\mathrm{kg} / \mathrm{m}^{3}\right)$ \\
\hline Arce & Acer pseudoplatanus L. & Aceraceae & Europa y oeste de Asia & 660 \\
\hline Maple & Acer rubrum L. & Aceraceae & Norteamérica & $460-510$ \\
\hline Pinabete & Picea abies L. H. Karst & Pinaceae & Europa & $460-510$ \\
\hline Ébano & Diospyros crassiflora Hiern & Ebenaceae & África & $900-1010$ \\
\hline Cedro & Cedrela odorata & Meliacea & América tropical & 340 \\
\hline Pino & Pinus sp. & Pinaceae & América & 376 \\
\hline
\end{tabular}

Fuente: Loewe y Subiri 1998, Sjöstedt 2012, Gutiérrez-Vázquez et al. 2012, Karinkanta 2014, AB TRÄTÄLJA sin fecha, Hernández-Maldonado y Sotomayor-Castellanos 2014, Weber 2005 
CUADRO III. MUESTREO DIAGNÓSTICO DE POLVOS DE MADERA EN CICLO DE $24 \mathrm{~h}$

\begin{tabular}{|c|c|c|}
\hline Taller & Día & Total de polvos $\left(\mathrm{mg} / \mathrm{m}^{3}\right)$ \\
\hline \multirow{4}{*}{1} & 1 & 1.47 \\
\hline & 2 & 1.46 \\
\hline & 3 & 2.18 \\
\hline & 4 & 2.89 \\
\hline \multicolumn{2}{|c|}{ Promedio } & 2.0 \\
\hline \multicolumn{2}{|c|}{ Desviación estándar } & 0.682 \\
\hline \multirow{4}{*}{2} & 1 & 2.88 \\
\hline & 2 & 2.16 \\
\hline & 3 & 1.44 \\
\hline & 4 & 2.16 \\
\hline \multicolumn{2}{|c|}{ Promedio } & 2.16 \\
\hline \multicolumn{2}{|c|}{ Desviación estándar } & 0.588 \\
\hline \multirow{4}{*}{3} & 1 & 2.16 \\
\hline & 2 & 2.16 \\
\hline & 3 & 1.49 \\
\hline & 4 & 1.55 \\
\hline \multicolumn{2}{|c|}{ Promedio } & 1.84 \\
\hline \multicolumn{2}{|c|}{ Desviación estándar } & 0.369 \\
\hline \multicolumn{2}{|c|}{ Promedio general } & 2.00 \\
\hline \multicolumn{2}{|c|}{ Desviación estándar general } & 0.526 \\
\hline
\end{tabular}

promedio de $1.47 \pm 0.18 \mathrm{mg} / \mathrm{m}^{3}$ en ocho horas. Este último promedio supera el límite de la norma francesa y canadiense que es de $1.0 \mathrm{mg} / \mathrm{m}^{3}$ (Cuadro IV).

\section{Encuesta}

La edad promedio de alumnos y profesores encuestados en los talleres de Laudería, es de 32.9 años, la edad mínima es de 21 y la máxima de 50 . La media de años trabajando la madera son 10.8 años, con un mínimo de uno y un máximo de 45 . La cantidad de horas en promedio, que los alumnos y profesores están en contacto directo con los polvos de la madera es de $5.7 \mathrm{~h}$ diarias, con un mínimo de dos y un máximo de 12 .

De los 20 usuarios que respondieron la encuesta, ocho mencionaron haber presentado alguna enfermedad relacionada con los ojos, nueve con la nariz, ocho con la garganta y seis con la piel.
CUADRO IV. MUESTREO DEFINITIVO DE POLVOS DE MADERAEN CICLO DE $4 \mathrm{~h}$ Y ESTIMACIÓN DE $8 \mathrm{~h}$

\begin{tabular}{|c|c|c|c|}
\hline Taller/Día & $\begin{array}{l}\text { Total de } \\
\text { polvos } \\
(\mu g)\end{array}$ & $\begin{array}{l}\text { Total de polvos } \\
\text { de madera, ciclo } \\
\text { de } 4 \mathrm{~h}\left(\mathrm{mg} / \mathrm{m}^{3}\right)\end{array}$ & $\begin{array}{c}\text { Estimación de polvos } \\
\text { de madera, estimación } \\
8 \mathrm{~h}\left(\mathrm{mg} / \mathrm{m}^{3}\right)\end{array}$ \\
\hline $1-1$ & 800 & 0.96 & 1.92 \\
\hline $1-2$ & 800 & 0.96 & 1.92 \\
\hline $1-3$ & 600 & 0.72 & 1.44 \\
\hline $1-4$ & 800 & 0.96 & 1.92 \\
\hline $1-5$ & 500 & 0.60 & 1.20 \\
\hline $1-6$ & 500 & 0.60 & 1.20 \\
\hline $1-7$ & 600 & 0.72 & 1.44 \\
\hline $1-8$ & 500 & 0.60 & 1.20 \\
\hline $1-9$ & 500 & 0.60 & 1.20 \\
\hline $1-10$ & 800 & 0.96 & 1.92 \\
\hline \multicolumn{2}{|c|}{ Promedio } & 0.77 & 1.54 \\
\hline \multicolumn{2}{|c|}{ Desviación estándar } & 0.17 & 0.34 \\
\hline $2-1$ & 600 & 0.72 & 1.44 \\
\hline $2-2$ & 500 & 0.60 & 1.20 \\
\hline $2-3$ & 600 & 0.72 & 1.44 \\
\hline $2-4$ & 500 & 0.60 & 1.20 \\
\hline $2-5$ & 700 & 0.84 & 1.68 \\
\hline $2-6$ & 600 & 0.72 & 1.44 \\
\hline $2-7$ & 600 & 0.72 & 1.44 \\
\hline $2-8$ & 600 & 0.72 & 1.44 \\
\hline $2-9$ & 600 & 0.72 & 1.44 \\
\hline $2-10$ & 700 & 0.84 & 1.68 \\
\hline \multicolumn{2}{|c|}{ Promedio } & 0.72 & 1.44 \\
\hline \multicolumn{2}{|c|}{ Desviación estándar } & 0.08 & 0.16 \\
\hline $3-1$ & 500 & 0.60 & 1.2 \\
\hline $3-2$ & 500 & 0.60 & 1.2 \\
\hline $3-3$ & 700 & 0.84 & 1.68 \\
\hline $3-4$ & 600 & 0.72 & 1.44 \\
\hline $3-5$ & 600 & 0.72 & 1.44 \\
\hline $3-6$ & 700 & 0.84 & 1.68 \\
\hline $3-7$ & 600 & 0.72 & 1.44 \\
\hline $3-8$ & 700 & 0.84 & 1.68 \\
\hline $3-9$ & 600 & 0.72 & 1.44 \\
\hline $3-10$ & 500 & 0.60 & 1.47 \\
\hline \multicolumn{2}{|c|}{ Promedio general } & 0.72 & 1.47 \\
\hline \multicolumn{2}{|c|}{ Desviación estándar } & 0.10 & 0.18 \\
\hline
\end{tabular}

De los 20, solamente ocho señalaron percibir una relación directa entre enfermedad y polvos de madera. Once encuestados indicaron usar alguno de los siguientes equipos de protección: mandil, mascarilla, guantes, tapones de oídos, lentes de protección o cubreboca.

Para 15 encuestados, los procesos que más generan polvos y otros residuos de madera fueron el lijado y para 11, el pulido. Finalmente, respecto al monitoreo periódico de la salud, los casos que indicaron haberse realizado análisis de audiometría fue uno, oftalmología tres, exploración cutánea uno y análisis general uno. 


\section{DISCUSIÓN}

Se reconoce que la presencia de polvos de madera en el aire del interior es un factor de riesgo laboral que afecta negativamente la salud humana debido a la composición química de dichos polvos, así como por ser un medio de transporte para bacterias y hongos (Gioffre et al. 2012). Internacionalmente se reconoce que los polvos de madera provocan enfermedades respiratorias alérgicas y no alérgicas, como son: asma no alérgica, rinitis, bronquitis crónica, obstrucción crónica, alveolitis alérgica extrínseca y cáncer, entre otras (Kenny et al. 1999, Schlünssen et al. 2001, Douwes et al. 2003, Ideara 2014).

La normatividad mexicana sobre los polvos de madera (Ley Federal del Trabajo, artículo 513, 2015) reconoce como enfermedades de trabajo las afecciones debidas su inhalación en carpinteros, madereros, ebanistas y trabajadores de la industria papelera. Se reconoce como límite máximo de exposición a polvos de madera dura $1 \mathrm{mg} / \mathrm{m}^{3}$ y de maderas suaves 5 $\mathrm{mg} / \mathrm{m}^{3}$ (STPS 1994). Por otro lado, la normatividad francesa y canadiense señala como límite de tolerancia $1 \mathrm{mg} / \mathrm{m}^{3}$, en Dinamarca y Suecia $2 \mathrm{mg} / \mathrm{m}^{3}$, en Finlandia y España $5 \mathrm{mg} / \mathrm{m}^{3}$ y en Estados Unidos de Norteamérica entre 5 y $15 \mathrm{mg} / \mathrm{m}^{3}$ (Bornholdt 2008).

En México no se han realizado estudios sistemáticos sobre la exposición a polvos de madera en talleres de construcción de instrumentos musicales. Y aunque este país alberga una de las pocas escuelas en el mundo que imparte una licenciatura en Laudería, no se garantiza la salud de alumnos y profesores en sus talleres.

El promedio de polvos de madera presentes en el aire del interior de los talleres de la Escuela de Laudería fue de $2.0 \pm 0.52 \mathrm{mg} / \mathrm{m}^{3}$ en $24 \mathrm{~h}, 0.74 \pm$ $0.10 \mathrm{mg} / \mathrm{m}^{3}$ en $4 \mathrm{~h}$ y como promedio estimado para $8 \mathrm{~h} 1.47 \pm 0.18 \mathrm{mg} / \mathrm{m}^{3}$. Estos resultados evidencian que la concentración de polvos de madera presentes en el aire del interior de los talleres de la Escuela de Laudería del INBAL rebasa el límite de las normas oficiales de Francia, Canadá, Dinamarca y Suecia, así como las españolas.

La concentración de polvos en los talleres de la escuela de laudería representa un riesgo para la salud de los usuarios, por lo que es necesario tomar medidas preventivas y correctivas, así como personales e institucionales para reducir su presencia en el aire del interior de los talleres de construcción de instrumentos musicales.

Es indispensable el uso de equipo de protección como lentes, mascarillas filtrantes para partículas y ropa de trabajo limpia (preferentemente de manga larga). El cabello debe estar recogido y cubierto mientras se trabaja con maderas que presentan sustancias dañinas (como quinonas y colofonia), algunos ejemplos son: el cedro rojo (Goldsmith y Shy 1988), ébano (Ideara 2014), arce y pinabete (Fengel y Wegener 1989). De la misma forma los alumnos y profesores deben protegerse de los polvos de polímeros. Cabe mencionar la importancia de usar equipo de seguridad complementario como guantes, mandil, entre otros.

Es necesario que la Escuela de Laudería del INBAL implemente medidas de seguridad en los talleres de construcción de instrumentos musicales, tales como extractores y purificadores de aire, bancos de trabajo con extractores y captadores de polvos, uso obligatorio de equipo de seguridad y fomento de la cultura del cuidado individual de la salud.

\section{CONCLUSIONES}

Los efectos de las partículas de madera sobre la salud humana son un fenómeno complejo, ya que no solamente implica la concentración, es decir, la cantidad de partículas en un determinado volumen de aire, sino también la frecuencia con que los polvos están presentes en el interior, la recurrencia con que el personal se expone a estas partículas, los tipos de madera y otros materiales como son metales y polímeros. Por otra parte están los aspectos relativos a la información sobre seguridad laboral, la cultura de la prevención y el cuidado individual de la salud.

Se puede señalar que el aire del interior en los talleres de la Escuela de Laudería del INBAL, México, no es de buena calidad porque en promedio presentaron una concentración superior a los $2 \mathrm{mg} / \mathrm{m}^{3}$ en $24 \mathrm{~h}$. Esto es un límite máximo señalado por la normatividad de Francia, Canadá, Suecia, Dinamarca, el Instituto Nacional de Seguridad e Higiene en el Trabajo de España (INSHT sin fecha) y el Centro Tecnológico del Mármol y la Piedra (2009). Asimismo no existe un reconocimiento generalizado por parte de alumnos y profesores, sobre la relación directa entre los polvos de la madera y la salud humana.

Por lo anterior, se concluye que los efectos de los polvos de la madera sobre la salud humana es un proceso sinérgico donde intervienen procesos laborales, materiales usados, cultura, información y cuidado individual de la salud, entre otros. Por ello las medidas para minimizar los efectos dañinos sobre la salud humana deben considerar estos y otros aspectos, ya que medidas o restricciones unilaterales no generarían una mejora significativa. 
Por otra parte se recomienda identificar y conocer con precisión las sustancias presentes en los extraíbles de las maderas, así como las bacterias y hongos que pudieran estar adheridos a los polvos de las mismas, para poder ofrecer una retroalimentación puntual a la institución y que pueda desarrollar programas de mejoramiento ambiental.

Al cuantificar los polvos de la madera en aire del interior se dispone de información para organizar procesos de mejora institucional. La información generada, deberá facilitar la toma de decisiones por parte de la institución en lo concerniente a la instalación de sistemas de ventilación y captura de polvos, el uso de equipo de protección personal y así minimizar el impacto que estos pudieran tener sobre la salud humana.

\section{AGRADECIMIENTOS}

Se agradece al Lic. Luis Gilberto Lavalle Guillén, las facilidades ofrecidas para el desarrollo de este estudio, a la Ing. Martha Laura Pérez Valdés, responsable del Laboratorio de Tecnología Ambiental de la Universidad Tecnológica de Querétaro, por su ayuda y facilidades para el uso de las instalaciones del mismo.

\section{REFERENCIAS}

AB TRÄTÄLJA (s/f). Spruce/whitewood (Picea abies). Information from your swedish timber exporter [en línea]. http://www.tratalja.se/ckfinder/userfiles/files/ Spruce_Whitewood_Picea\%20abies.pdf

Airmetrics (2012). MiniVol tactical air sampler, Airmetrics operation manual. Rev. 1.2. Eugene, Oregon, EUA, 75 pp.

Bornholdt L.J. (2008). Effects of wood dust: inflamation, genotoxicity and cáncer. Tesis doctoral. National Research Centre for The Working Environment. Faculty of Health Sciences. Copenhagen, Dinamarca, 78 pp.

Catalán-Vázquez M. y Jarillo-Soto E. (2010). Paradigmas de investigación aplicados al estudio de la percepción pública de la contaminación del aire. Rev. Int. Contam. Ambie. 26, 165-178.

Centro Tecnológico del Mármol y la Piedra (2009). El polvo de la madera: riesgo laboral y su prevención. Federación de Industria, Madrid, España, 130 pp.

Douwes L., Thorne P., Pearce N. y Heederik D. (2003). Bioaerosol healt effects and exposure assessment: progress and prospects. Ann. Occup. Hyg. 47, 187-200. DOI: $10.1093 /$ annhyg/meg032

Echenique-Manrique R. y Robles F.F. (1993). Ciencia y tecnología de la madera tomo I. Universidad Veracruzana, Xalapa, Veracruz, México. 134 pp.
Fengel D. y Wegener G. (1989). Wood: chemistry, ultrastructure, reactions. De Gruyter, Berlin-Nueva York, EUA, 613 pp.

Gioffre A., Marramao A. e Ianno A. (2012). Airborne microorganisms, endotoxinas, and dust concentration in wood factories in Italy. Ann. Occup. Hyg. 5,161-169. DOI: 10.1093/annhyg/mer082

Goldsmith D.F. y Shy C.M. (1988). Respiratory health effects from occupational exposure to wood dust. Scandinavian Journal of Work, Enviromental and Healt 14, 1-15. DOI: 10.5271/sjweh. 1958

Gutiérrez-Vázquez B.N., Cornejo-Oviedo E.H., GutiérrezVázquez M.H. y Gómez-Cárdenas M. (2012). Variación y predicción de la densidad básica de la madera de Cedrela odorata L. Rev. Fitotec. Mex. 35, 87-90.

Hernández-Maldonado S. A. y Sotomayor-Castellanos J. R. (2014). Comportamiento elástico de la madera de Acer rubrum y de Abies balsamea. Madera y Bosques 20, 113-123.

Ideara S.L. (2014). Estudio, análisis y consecuencias del uso de agentes químicos y maderas en los luthiers de la comunidad de Madrid, Instituto Regional de Seguridad y Salud en el Trabajo, Madrid, España, 71 pp.

INSHT (sin fecha). Norma Oficial MTA/MA-014/A11. Determinación de materia particulada (fracción inhalable, torácica y respirable) en aire. Método gravimétrico. Instituto Nacional de Seguridad e Higiene en el Trabajo. Madrid, España, 17 pp.

Karinkanta P. (2014). Dry fine grinding of Norway spruce (Picea abies) wood in impact-based fine grinding mills. Tesis Doctoral. Acta Universitatis Ouluensis C 516. University of Oulu, Finlandia, 107 pp.

Kauppinen T., Vincent R., Liukkonen T., Grzebyk M., Kauppinen A., Welling I., Arezes P., Black N., Bochmann F., Campelo F., Costa M., Elsigan G., Goerens R., Kikemenis A., Kromhout H., Miguel S., Mirabelli D., Mceneany R., Pesch B., Plato N., Schlünssen V., Schulze J., Sonntag R., Verougstraete V., De Vicente M.A., Wolf J., Zimmermann M., Husgafvel-Pursiainen K. y Savolainen K. (2006). Occupational exposure to inhalable wood dust in the member state of the European Union. Ann. Occup. Hyg. 50, 549-561.

DOI: 10.1093/annhyg/mel013

Kenny L.C., Bowry A., Crook B. y Stancliffe J. D. (1999). Field testing of personal size-selective bioaerosol sampler. Ann. Occup. Hyg. 43, 393-404.

DOI: 10.1093/annhyg/43.6.393

Kochevar S.D. (2006). Basic guide to particle counters and particle counting. Particle Measuring Systems, Inc. Colorado, EUA, 54 pp.

Lavalle G. L. G. 2015. La Laudería en México. En: Los sonidos de la madera: Los árboles de México y su relación con la creación de instrumentos musicales 
tradicionales y clásicos (J. A. Quiroz Carranza y C. Cantú Gutiérrez, Ed.). Plaza y Valdés. Ciudad de México, México, pp. 167-182.

Ley Federal del Trabajo (2015). Última reforma publicada el 12 de junio de 2015, Ciudad de México, México, 236 pp.

Loewe M.V. y Subiri P.M. (1998). Sicomoro. Acer pseudoplatanus. Monografía. Instituto Forestal. Santiago de Chile, Chile, $42 \mathrm{pp}$.

Magagnotti N., Nannicini C., Sciarra G., Spinelli R. y Volpi D. (2013). Determining the exposure of chiper operators to inhalable Wood dust. Ann. Occup. Hyg. 57, 784-792. DOI: 10.1093/annhyg/mes 112

Meo S. A. (2004). Effects of duration of exposure to wood dust on peak expiratory flow rate among workers in small scale wood industries. Int. J. Occup. Med. Environ. Healt 17, 451-455.

STPS (1994). Norma Oficial Mexicana NOM-010STPS-1994. Relativa a las condiciones de seguridad e higiene en los centros de trabajo donde se produzcan, almacenen o manejen sustancias químicas capaces de generar contaminación en el medio ambiente laboral. Secretaría del Trabajo y Previsión Social. Diario Oficial de la Federación. 26 de octubre de 1993.
Schlünssen V., Venzents P.S., Mikkelsen A. B. y Schaumburg I. (2001). Wood dust exposure in the danish forniture industry using conventional and passive monitors. Ann. Occup. Hyg. 45, 157-164. DOI: $10.1016 / \mathrm{S} 0003-4878(00) 00055-7$

Sjöstedt J. (2012). A literature study and survey of sycamore maple (Acer pseudoplatanus L.) in southern Sweden. Tesis de maestría. Swedish University of Agricultural Sciences, Alnarp, Suecia, 53 pp.

Teschke K., Demers P.A., Davies H.W., Kennedy S.M., Marion S. A. y Leung V. (1999). Determinants of exposure to inhalable particulate wood dust, resin acid, and monoterpenes in lumber mill environment. Ann. Occup. Hyg. 43, 247-255.

DOI: 10.1093/annhyg/43.4.247

Tzintzun C. Ma. G., Roja B. L. y Fernández B. A. (2005). Las partículas suspendidas en tres grandes ciudades mexicanas. Gaceta Ecológica 74,15-28.

Weber E.M. (2005). Densidad de madera de Pinus taeda L. Marion de diferentes edades, Misiones, Argentina. Revista Floresta 35, 487-494

DOI: $10.5380 /$ rf.v35i3.5199 